\title{
Towards a Transdisciplinary Method: Teaching and research practices in immersive spaces and mixed realities
}

\author{
Julian Stadon \\ University of Hertfordshire \\ UK \\ julianstadon@gmail.com
}

\section{INTRODUCTION}

This paper acts as a provocation for a panel that will take place this year at the EVA conference. The panel brings together a number of UK based academics and institutions. The field of immersive mixed reality traverses a diverse range of fields and industries and due to this, traditional methods for teaching and research have to be reoriented towards a more collaborative, transdisciplinary approach, without evolving at the expense of skills learning, industry awareness, employability, specialisation and quality of outcomes produced.

\section{TRANS VS CROSS-DISCIPLINARY COLLABORATION}

As cultural producers, researchers and educators, we need to be weaving humanity into the fabric of digital media. This can only be achieved through educating transdisciplinary approaches, particularly in the scope of tertiary education. The problem is that within most university structures, pedagogical approaches are traditionally structured to be paradigmatic within particular fields, generally with a clear intention to train students for specific industries.

Real collaboration can only exist when all parties understand the foundations of each other's field, which is a very difficult teaching goal to achieve within a three or four-year timeframe. The old artisanal model of art and design schools, where each student focuses on becoming professionally proficient in a single chosen field of speciality is no longer a model to work by in this participatory, convergent, contemporary media landscape. The metaphor of lifelong learning and flexible ontologies towards learning and disciplines is more than just rhetoric and with the advent of Open Design, our pedagogical approaches much shift accordingly.
Traditional craft-based approaches to technology produce objects of value. Today consumers are much more interested in the value of experience and this is one way that digital technology has redefined our approaches to making.

This is directly relational to mixed realty and immersion, because the reductive nature of (interface) design often obfuscates our embodied interactions with technology. In VR, AR and MR/XR we see a shift towards a direct engagement with particular tools and virtual craftsmanship. Google Tilt Brush for example, demonstrates this return to a more gestural interaction with digital space, through reorienting the screen-based interfaces that we access it through. The real-time human pose recognition and alignment of the Leap Motion and Kinect, allowing for natural gestural interactions and computer vision based immersion are also examples of this.

There is an emergent trend of playful and functional involvement in embodiment within production, which could in fact be said to be a return to tradition methods of craftsmanship using hands and bodyembodied production rather than a Manovich paradigm of software taking command.

\section{OPEN DESIGN STRATEGIES FOR TRANSDISCIPLINARY EDUCATION}

The UK invests £6bn per year less that both France and Germany into employee training and life long learning for employed individuals. That has fallen $£ 5.1$ bn since 2010 (McNeil \& Dowler 2017). Open Design and Rapid Prototyping Methods are already replacing traditional skills based digital learning within art and design education, and this should be recognised and articulated more clearly within course curriculums and descriptions. It is often the case that students in the UK pay for what they 
expect to be a technical instruction based course, when in most cases it is much more complex and nuanced towards a wider ranges of learning pathways and therefore potentially more vague in their approach in the eyes of students. The goal really is to educate a commitment to independent learning of skills, in order to also address the new spatial and cultural contexts of immersive media.

This is achieved through three convergent methods:

1. Using innovative digital platforms that are embedded within participatory culture, designed for digital natives and utilise emerging technologies to enable you to transfer content in a more innovative way.

2. Developing a critical understanding of media technologies within practice, industry and society.

3. Demystifying technology through the understanding of technological processes.

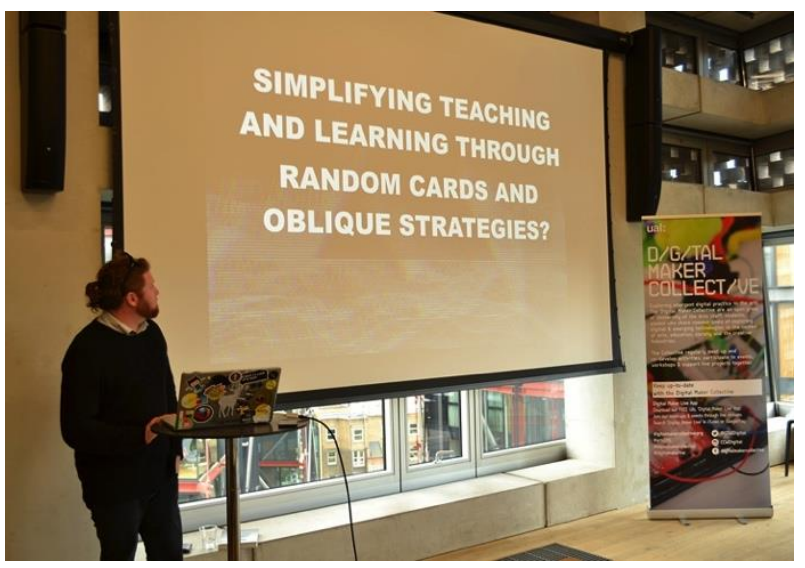

Figure 1: A transdisciplinary intervention.

\section{PANEL GOALS AND INTENTIONS}

This panel will discuss historical and current approaches to educating in the fields of immersive environment based art and design, while also speculating how to integrate flexible pedagogical approaches within such scenarios. This will include a scoping study of UK-based tertiary programmes, along with an open discussion of topics including:

- Bridging theory and practice.

- Developing a theoretical framework for teaching IMR that builds on historical achievements and acknowledges several directions for the future.

- Integrating theory specific to immersion and reality into existing theory pedagogy. Adapting and extending tools and techniques from precursor creative practice pedagogy.

- Teaching and researching beyond evangelical approaches and shallow trends.

- Scoping and defining transdisciplinary approaches that build momentum and accelerate wider adoption.

- Negotiating Industry Integration into teaching and collaborative research.

- Driving the convergence of entertainment and more serious industry driven projects.

- Commercialisation of outcomes and IP issues.

- Methods for collaboration across disciplines

- Where to situate teaching and research: studio, lab or classroom?

- Ethical considerations and User Centred Design.

The discussion of these topics aims to identify commonalities in approaches and explore current research and state of the art technologies that exist today, fostering new and lasting connections within the field and tackling current and future obstacles facing its widespread adoption. This discussion will then transition into an open forum, which encourages audience participants to also engage in these core issues relating to the topic, in order to widen opportunities for learning and networking within creative and adjacent fields of immersive technology.

\section{REFERENCES}

McNeil, C. and Dowler, R. (2017) Can Skills Survive the Robot Revolution? https://www.newstatesman.com/microsites/skills/20 17/07/can-skills-survive-robot-revolution (retrieved 26 March 2018). 